\title{
Sesamoid bone interposition in the interphalangeal joint after dislocation of the hallux: A case report
}

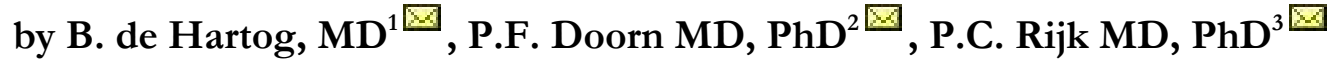

The Foot and Ankle Online Journal 2 (7): 3

A 39 year-old woman injured the big toe of her right foot after a fall down the stairs. Upon examination there was a dislocation of the hallux. A plain radiograph showed a dislocation of the interphalangeal joint of the hallux and interposition of a sesamoid bone in the articular space. An attempt at closed reduction was unsuccessful, after which open reduction and excision of the sesamoid was undertaken. Dislocation of the interphalangeal joint of the hallux with interposition of a sesamoid is a rare injury. It is almost always the result of a hyperextension trauma. The clinical appearance together with a plain radiograph is in most cases sufficient for the diagnosis. Closed reduction should be attempted before open reduction is undertaken. If this is unsuccessful, it is probably due to an invaginated volar plate and sesamoid. Open reduction and removal of the sesamoid is then required followed by reduction of the volar plate plantarward. In general, the recovery is complete and without problems.

Keywords: Dislocation, hallux, interposition, sesamoids, treatment

Accepted: June, 2009

Published: July, 2009

This is an Open Access article distributed under the terms of the Creative Commons Attribution License. It permits unrestricted use, distribution, and reproduction in any medium, provided the original work is properly cited. @The Foot and Ankle Online Journal (www.faoj.org)

A

dislocation of the hallux with interposition of the sesamoid bone is a rare injury. ${ }^{1,2,3,4,5,6}$ The

treatment can consist of a closed reduction or an open repair. We report a 39 year-old woman with a painful hallux after a fall down the stairs. On the initial radiographs interposition in the interphalangeal joint (IPJ) of a sesamoid bone was seen. An attempt at closed reduction was unsuccessful after which the patient was treated with open reduction and excision of the sesamoid.

\footnotetext{
Address correspondence to: P.C. Rijk, P.O. Box 888, 8901 BR Leeuwarden, The Netherlands, Tel: 0031-582867282, Fax: 0031-582867611

Email: P.Rijk@znb.nl

1,2,3 Bas de Hartog M.D, Peter F. Doorn M.D, PhD, Paul C. Rijk M.D, PhD Department of Orthopaedic Surgery, Medical Centre Leeuwarden, Leeuwarden, The Netherlands.
}

\section{Case Report}

A 39 year-old woman injured her right hallux after a fall down the stairs. She presented at the emergency room with an abnormal configuration of the hallux and in a significant amount of pain. On physical examination there was a dorsal dislocation of the IPJ of the great toe. Marked swelling and tenderness of the hallux was also noted.

Passive movement of the toe was very painful, active movement was not possible. The neurovascular status of the hallux was normal. A plain radiograph of the hallux showed a dislocation of the IPJ of the hallux and an entrapment of a sesamoid bone in the interphalangeal space (Figs. 1A and 1B). After local anaesthesia an attempt at closed reduction was undertaken, but was unsuccessful. 

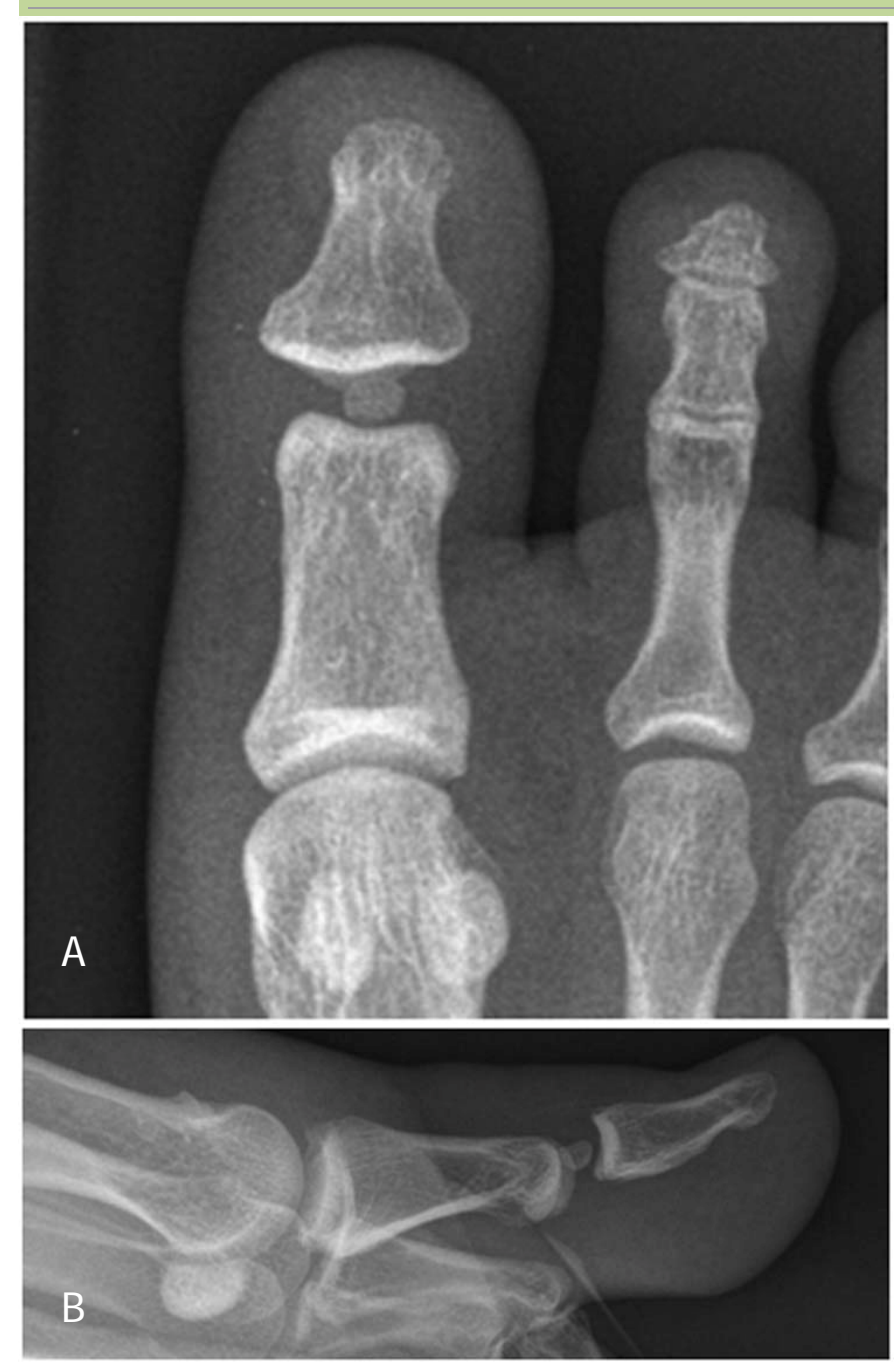

Figure 1A and 1B Radiograph of the right hallux showing the enlarged joint space with the entrapment of the sesamoid in the interphalangeal space. (A) anteroposterior view. Lateral view of the interphalangeal joint. (B)

Following this, surgical removal of the sesamoid and reduction of the hallux was undertaken. Under general anaesthesia, a dorsal S-type incision was made over the IPJ. The extensor tendon was incised longitudinally and the joint capsule was opened. The sesamoid together with the volar plate were found within the articular space. The sesamoid was removed and the volar plate was reduced from the IPJ after which the hallux could be reduced. The joint capsule was closed and the skin was approximated.
The patient was treated with 2 days compression bandage and normal mobilisation. The follow up was uneventful and the recovery was complete.

\section{Discussion}

Sesamoid bones of the hallux develop in the fetal period and aid the biomechanics of joint function. ${ }^{7}$ The prevalence of interphalangeal sesamoids is approximately $13 \% .^{8}$ The hallux can have one or two interphalangeal sesamoids. They are integrated in the plantar plate, which consist of the flexor hallucis longus tendon and the plantar joint capsule. ${ }^{1,4,8}$ Dislocation of the IPJ with entrapment of a sesamoid is a rare injury and has not often been described in the literature. ${ }^{1,2,3,4}$ This is partly due to tough soft tissue structures, which prevent hyperextension of the toe. Only when the collateral ligaments, the capsule and the volar plate are injured a dislocation of the IPJ is possible..$^{5}$ Often a hyperextension or an acute axial load type injury precedes an IPJ dislocation., ${ }^{4,9}$ Muller described this type of injury for the first time in the Lancet in $1944 .^{10}$ At first presentation there is often tenderness of the hallux with inability of active movement. Physical examination involves palpation of the hallux, metatarsal phalangeal joint and sesamoid complex. A plain anteroposterior radiograph of the hallux is in most cases sufficient for the diagnosis. A widened interphalangeal space and interposition of a sesamoid has been observed. In some cases an overlapping of the two phalangeal bones with narrowing of the interphalangeal space can be seen. On the other hand, it has to be recognised that an epiphysial injury in adolescents can mimic a sesamoid dislocation. ${ }^{2}$ Treatment can consist of closed reduction or surgical treatment. ${ }^{2,8,11}$ Closed reduction, which has been successful in some cases, should be attempted by giving longitudinal traction, dorsal flexion follow by fast flexion in the IPJ. However, in several case reports it has been demonstrated that closed reduction is not successful. In these cases open reduction and reflexion or removal of the interphalangeal sesamoid and/or volar plate plantarward is mandatory. Although removal might have a negative effect on the biomechanics of the hallux, in most cases a complete recovery is made. ${ }^{8}$ 


\section{References}

1. Eibel P: Dislocation of the interphalangeal joint of the big toe with interposition of a sesamoid bone. J Bone Joint Surg 36A (4): 880 - 882, 1954.

2. Dave D, Jayaraj VP, James SE: Intra-articular sesamoid dislocation of the interphalangeal joint of the great toe. Injury 24 (3): 198 - 199, 1993

3. Berger JL, LeGeyt MT, Ghobadi R: Incarcerated subhallucal sesamoid of the great toe: Irreducible dislocation of the great toe by an accessory sesamoid bone. Am J Orthop 26: 116 - 228, 1997.

4. Sorene E.D, Regev G: Complex dislocation with double sesamoid entrapment of the interphalangeal joint of the hallux. Foot Ankle Surg 46 (6): 413 - 416, 2006.

5. Miki T, Yamamuro T, Kitai T: An irreducible dislocation of the great toe. Report of two cases and review of the literature. Clin Orthop Relat Res 230 : 200 - 206, 1988

6. Kursunuglu S, Resnick D, Goergen T: Traumatic dislocation with sesamoid entrapment in the interphalangeal joint of the great toe. J Trauma $27: 959$ - 961, 1987.

7. Rodeo SA, Warren RF, O'Brien SJ, Pavlov H: Diastasis of bipartite sesamoids of the first metatarsal phalangeal joint. Foot Ankle 14: 425 - 434, 1993.

8. Davies MB Abdlslam K, Gibson RJ: Interphalangeal sesamoid bones of the great toe: An anatomic variant demanding careful scrutiny on radiographs. Clinical anatomy 16: $520-521$, 2003.

9. Nelson TL, Uggen W: Irreducible dorsal dislocation of the interphalangeal joint of the great toe. Clin Orthop Relat Res 157: $110-112,1981$.

10. Müller G.M: Dislocation of sesamoid of Hallux. Lancet 1: 789, 1944.

11. Szucs R, Hurwitz J: Traumatic subluxation of the interphalangeal joint of the hallux with interposition of the sesamoid bone. Am J Roentgenol 152 : 652 - 653, 1989. 